International Mathematical Forum, Vol. 9, 2014, no. 10, 457 - 465

HIKARI Ltd, www.m-hikari.com

http://dx.doi.org/10.12988/imf.2014.39175

\title{
On Subgroups of Quasi-Graph Groups
}

\author{
R. M. S. Mahmood \\ Irbid National University, P. O. Box 2600-Zip Code 21110, Irbid, Jordan.
}

Copyright (C) 2014 R. M. S. Mahmood. This is an open access article distributed under the Creative Commons Attribution License, which permits unrestricted use, distribution, and reproduction in any medium, provided the original work is properly cited.

\begin{abstract}
A graph is called a quasi-graph if the case of an edge of the graph equals its inverse is allowed. A graph of groups is called a quasi-graph of groups if the corresponding graph is a quasi-graph. A group is termed quasi-graph group if it is a fundamental group of a non-trivial quasi-graph of groups. In this paper we show that a subgroup of a quasi-graph group is a quasi-graph group.
\end{abstract}

Mathematics Subject Classification: 20E08, 20F05, 20E06

Keywords: quasi-graphs of groups, groups acting on trees

\section{Introduction}

In [4], Mahmood introduced the concepts of quasi-graphs of groups and their fundamental groups. The main result of this paper is to show that if $G$ is a fundamental group of a quasi-graph of groups and if $\mathrm{H}$ is a subgroup of $\mathrm{G}$, then we use the results of [5] and [6] to show that $\mathrm{H}$ is a fundamental group of a quasigraph of groups. This paper is divided in to 4 sections. In section 2, we introduce the concepts quasi graphs, groups acting on trees with inversions, and their fundamental domains. In section 3, we use the results of section 2 to obtain the structures of quasi-graphs of groups induced by the fundamental domains for groups acting on trees with inversions. In section 4, we apply the results of section 3 to obtain the structures of quasi-graphs of groups induced by the subgroups of the fundamental groups of quasi-graphs of groups. 


\section{Quasi-Graphs Induced by Fundamental Domains}

A quasi graph $X$ consists of two disjoint sets $V(X)$, (the set of vertices of $X$ ) and $\mathrm{E}(\mathrm{X})$, (the set of edges of $\mathrm{X}$ ), with $\mathrm{V}(\mathrm{X})$ non-empty, together with three functions $\partial_{0}: \mathrm{E}(\mathrm{X}) \rightarrow \mathrm{V}(\mathrm{X}), \partial_{1}: \mathrm{E}(\mathrm{X}) \rightarrow \mathrm{V}(\mathrm{X})$, and $\eta: \mathrm{E}(\mathrm{X}) \rightarrow \mathrm{E}(\mathrm{X})$ is an involution satisfying the conditions that $\partial_{0} \eta=\partial_{1}$ and $\partial_{1} \eta=\partial_{0}$. For simplicity, if e $\in \mathrm{E}(\mathrm{X})$, we write $\partial_{0}(\mathrm{e})=\mathrm{o}(\mathrm{e}), \partial_{1}(\mathrm{e})=\mathrm{t}(\mathrm{e})$, and $\eta(\mathrm{e})=\bar{e}$. This implies that $\mathrm{o}(\bar{e})=\mathrm{t}(\mathrm{e})$, $\mathrm{t}(\bar{e})=\mathrm{o}(\mathrm{e})$, and $\overline{\bar{e}}=\mathrm{e}$. The case $\bar{e}=\mathrm{e}$ is allowed. There are obvious definitions of subgraphs, circuits, trees, morphisms of graphs and Aut $(\mathrm{X})$, the set of all automorphisms of the graph $\mathrm{X}$ which is a group under the composition of morphisms of graphs. For more details, the interested readers are referred to in [1], [2] and [9]. We say that a group $G$ acts on a graph $X$ if there is a group homomorphism $\phi: G \rightarrow \operatorname{Aut}(X)$. In this case, if $x \in X$ (vertex or edge) and $g \in G$, we write $\mathrm{g}(\mathrm{x})$ for $(\phi(\mathrm{g}))(\mathrm{x})$. Thus, if $\mathrm{g} \in \mathrm{G}$, and $\mathrm{y} \in \mathrm{E}(\mathrm{X})$, then $\mathrm{g}(\mathrm{o}(\mathrm{y}))=\mathrm{o}(\mathrm{g}(\mathrm{y}))$, $\mathrm{g}(\mathrm{t}(\mathrm{y}))=\mathrm{t}(\mathrm{g}(\mathrm{y}))$, and $\mathrm{g}(\bar{y})=\overline{g(y)}$. The case the action with inversion is allowed. That is; $\mathrm{g}(\mathrm{y})=(\bar{y})$ is allowed for some $\mathrm{g} \in \mathrm{G}$, and $\mathrm{y} \in \mathrm{E}(X)$. In this case we say that $\mathrm{g}$ is an inverter element of $\mathrm{G}$ and $\mathrm{y}$ is called an inverted edge. If the group $\mathrm{G}$ acts on the graph $\mathrm{X}$ and $\mathrm{x} \in \mathrm{X}$, ( $\mathrm{x}$ is a vertex or edge), then

1. The stabilizer of x, denoted $G_{x}$ is defined to be the set $G_{x}=\{\mathrm{g} \in \mathrm{G}: \mathrm{g}(\mathrm{x})=\mathrm{x}\}$. It is clear that $G_{x} \leq \mathrm{G}$, and if $\mathrm{x} \in \mathrm{E}(\mathrm{X})$, and $\mathrm{u} \in\{\mathrm{o}(\mathrm{x}), \mathrm{t}(\mathrm{x})\}$, then $G_{\bar{x}}=G_{x}$ and $G_{x} \leq G_{u}$. 2. The orbit of $x$ is the set $G(x)=\{g(x): g \in G\}$. It is clear that $G$ acts on the graph $X$ without inversions if and only if $\mathrm{G}(\bar{e}) \neq \mathrm{G}(\mathrm{e})$ for any $\mathrm{e} \in \mathrm{E}(\mathrm{X})$.

3. The set of orbits is denoted by $G / X=\{G(x): x \in X\}$. In [6, Prop. 2.1], it is proved that $G / X$ forms a quasi-graph.

Definition. Let $\mathrm{G}$ be a group acting on a connected quasi-graph $\mathrm{X}$ with inversions, and let $\mathrm{T}$ and $\mathrm{Y}$ be two subtrees of $\mathrm{X}$ such that $\mathrm{T} \subseteq \mathrm{Y}$, and each edge of $\mathrm{Y}$ has at least one end in $\mathrm{T}$. Assume that $\mathrm{T}$ and $\mathrm{Y}$ are satisfying the following. (i) $\mathrm{T}$ contains exactly one vertex from each vertex orbit.

(ii) Y contains exactly one edge $\mathrm{y}$ (say) from edge orbit if $\mathrm{G}(\mathrm{y}) \neq \mathrm{G}(\bar{y})$ and exactly one pair $\mathrm{x}, \bar{x}$ from each edge orbit if $\mathrm{G}(\mathrm{x})=\mathrm{G}(\bar{x})$. Then

(1) $\mathrm{T}$ is called a tree of representatives for the action of $\mathrm{G}$ on $\mathrm{X}$,

(2) $\mathrm{Y}$ is called a transversal for the action of $\mathrm{G}$ on $\mathrm{X}$.

For simplicity we say that $(\mathrm{T} ; \mathrm{Y})$ is a fundamental domain for the action of $\mathrm{G}$ on $\mathrm{X}$. For more details, the readers are referred to [3]. The properties of fundamental domains for the actions of groups on connected quasi-graphs imply that if $\mathrm{G}$ is a group acting on a connected quasi-graph $\mathrm{X}$ with inversions, and $(\mathrm{T} ; \mathrm{Y})$ is a fundamental domain for the action of $G$ on $X$, then for any $v \in V(Y)$ and any 
$\mathrm{y} \in \mathrm{E}(\mathrm{Y})$, there exists a unique vertex denoted $\mathrm{v}^{*}$ of $\mathrm{T}$ such that $\mathrm{g}\left(\mathrm{v}^{*}\right)=\mathrm{v}$; that is, $\mathrm{G}\left(\mathrm{v}^{*}\right)=\mathrm{G}(\mathrm{v})$, and element denoted $[\mathrm{y}]$ of $\mathrm{G}$ satisfying the follows.

(a) if $\mathrm{o}(\mathrm{y}) \in \mathrm{V}(\mathrm{T})$, then $[\mathrm{y}]\left((\mathrm{t}(\mathrm{y}))^{*}\right)=\mathrm{t}(\mathrm{y}),[\mathrm{y}]=1$ in case $\mathrm{y} \in \mathrm{E}(\mathrm{T})$, and $[\mathrm{y}](\mathrm{y})=\bar{y}$ if $\mathrm{G}(\mathrm{y})=\mathrm{G}(\bar{y})$,

(b) if $\mathrm{t}(\mathrm{y}) \in \mathrm{V}(\mathrm{T})$, then $[\mathrm{y}](\mathrm{o}(\mathrm{y}))=(\mathrm{o}(\mathrm{y}))^{*},[y]=[\bar{y}]^{-1}$ if $\mathrm{G}(\mathrm{y}) \neq \mathrm{G}(\bar{y})$, and $[y]=[\bar{y}]$ if $\mathrm{G}(\mathrm{y})=\mathrm{G}(\bar{y})$.

If $\mathrm{y} \in \mathrm{E}(\mathrm{Y})$, let $[y][\bar{y}]=\partial_{y}$ and let $+\mathrm{y}$ be the edge $+\mathrm{y}=\mathrm{y}$ if $\mathrm{o}(\mathrm{y}) \in \mathrm{V}(\mathrm{T})$, and

$+\mathrm{y}=[\mathrm{y}](\mathrm{y})$ if $\mathrm{t}(\mathrm{y}) \in \mathrm{V}(\mathrm{T})$. It is clear that $\partial_{y}=1$ if $\mathrm{G}(\mathrm{y}) \neq \mathrm{G}(\bar{y})$, and $\partial_{y}=[y]^{2}$ if

$\mathrm{G}(\mathrm{y})=\mathrm{G}(\bar{y})$. Furthermore, $\mathrm{o}(+\mathrm{y})=(\mathrm{o}(\mathrm{y}))^{*}$, and $G_{+y} \leq G_{(o(y))^{*}}$ and if $\mathrm{G}(\mathrm{y})=\mathrm{G}(\bar{y})$

or $\mathrm{y} \in \mathrm{E}(\mathrm{T})$, then $G_{+y}=G_{y}$.

The main result of this section is the following lemma.

Lemma 2.1. Let $G$ be a group acting on a connected quasi-graph $X$ with inversions, and let $(\mathrm{T} ; \mathrm{Y})$ be a fundamental domain for the action of $\mathrm{G}$ on $\mathrm{X}$. Let $G /(X ; Y ; T)=\{G(v): v \in V(T)\} \cup\{G(e): e \in E(Y)\}$ and

$G /(X ; T ; T)=\{G(v): v \in V(T)\} \cup\{G(e): e \in E(T)\}$. Then $G /(X ; Y ; T)$ forms a connected quasi-graph and $G /(X ; T ; T)$ is a maximal subtree of $G /(X ; Y ; T)$.

Proof. Consider the sets $V(G /(X ; Y ; T))=\{G(v): v \in V(T)\}$ and $E(G /(X ; Y ; T))=\{G(e): e \in E(Y)\}$. Since $\mathrm{V}(\mathrm{X}) \neq \varnothing$, therefore $V(G /(X ; Y ; T)) \neq \varnothing$. The fact that for $\mathrm{v} \in \mathrm{V}(\mathrm{X})$ and $\mathrm{e} \in \mathrm{E}(\mathrm{X})$, the orbits $\mathrm{G}(\mathrm{v})$ and $\mathrm{G}(\mathrm{e})$ are disjoint implies that $V(G /(X ; Y ; T)) \cap E(G /(X ; Y ; T))=\varnothing$. For $\mathrm{e} \in \mathrm{E}(\mathrm{Y}), \mathrm{G}(\mathrm{e}) \in E(G /(X ; Y ; T))$, define $\mathrm{o}(\mathrm{G}(\mathrm{e}))=\mathrm{G}\left((\mathrm{o}(\mathrm{e}))^{*}\right), \mathrm{t}(\mathrm{G}(\mathrm{e}))=\mathrm{G}\left((\mathrm{t}(\mathrm{e}))^{*}\right)$, and $\overline{G(e)}=G(\bar{e})$. Then $G /(X ; Y ; T)$ forms a graph. Similar to the proof of Prop. 2.10f [6] we can show that $G /(X ; Y ; T)$ forms a connected quasi-graph.

Since $V(G /(X ; T ; T))=V(G /(X ; Y ; T))$, [ see Prop. 11 of 9], therefore $G /(X ; T ; T)$ is a maximal subtree of $G /(X ; Y ; T)$. This completes the proof.

Note. $G /(X ; Y ; T)$ is called the quotient graph induced by the fundamental domain $(\mathrm{T} ; \mathrm{Y})$ for the action of $\mathrm{G}$ on $\mathrm{X}$.

\section{Quasi-graphs of groups Induced by Fundamental Domains}

The concepts of quasi-graphs of groups and their fundamental groups introduced in [4] are modified and defined as follows.

A quasi-graph of groups is defined to be a pair $\Phi=(Z ; \Gamma)$ where $Z$ is a connected quasi-graph and $\Gamma$ is a mapping from $Z$ into the class of all groups; where the 
image of each element (vertex or edge) $x \in Z$ under $\Gamma$ is denoted by $\Gamma_{x}$.i.e. $\Gamma(x)=\Gamma_{x}$ such that for each edge $\mathrm{e} \in \mathrm{E}(\mathrm{Z})$ the following hold.

(1) $\Gamma_{\bar{e}}=\Gamma_{e}$;

(2) There exist monomorphisms denoted $\lambda_{e}: \Gamma_{e} \rightarrow \Gamma_{t(e)}$ and $\lambda_{\bar{e}}: \Gamma_{e} \rightarrow \Gamma_{o(e)}$;

(3) There exists an element denoted $\delta_{e} \in \Gamma_{e}$ such that $\delta_{e}=1$ if $\bar{e} \neq e$;

(4) There exists an automorphism $\mu_{e}: \Gamma_{e} \rightarrow \Gamma_{e}$ satisfying the conditions that $\mu_{e}\left(\delta_{e}\right)=\delta_{e}, \mu_{e}(a)=a$ if $\bar{e} \neq e$, and $\mu_{e}^{2}(a)=\delta_{e} a \delta_{e}^{-1}$ for all $a \in \Gamma_{e}$.

Note. For simplicity, we write $\Phi(Z)$ to mean $\Phi=(Z ; \Gamma)$, the quasi graph of groups.

Notation. Given the quasi-graph of groups $\Phi=(Z ; \Gamma)$. For the edge e $\in \mathrm{E}(Z)$ and the element $a \in \Gamma_{e}$. We have the following notation.

(1) Let $\lambda_{e}\left(\Gamma_{e}\right)=\Gamma^{e}, \lambda_{\bar{e}}\left(\Gamma_{e}\right)=\Gamma^{\bar{e}}$ and let $\lambda_{e}(a)=a^{e}$. It is clear that $\Gamma^{e} \leq \Gamma_{t(e)}$, $\Gamma^{\bar{e}} \leq \Gamma_{o(e)}, a^{e} \in \Gamma^{e}$, and $\lambda_{e}\left(\delta_{e}\right)=\delta_{e}^{e} \in \Gamma^{e}$.

(2) Let $\phi_{e}: \Gamma^{e} \rightarrow \Gamma^{\bar{e}}$ be the mapping given by $\phi_{e}\left(a^{e}\right)=\left(\mu_{e}(a)\right)^{\bar{e}}$. It is clear that $\phi_{e}$ is an isomorphism and if $\bar{e} \neq e$ then $\phi_{e}\left(a^{e}\right)=a^{\bar{e}}$.

Definition. Given the quasi-graph of groups $\Phi(Z, \Gamma)$. Let $\Lambda$ be a maximal subtree of Z. Define $\pi \Phi(Z ; \Gamma ; \Lambda)$ to be the group of the presentation: $\left\langle\operatorname{gen}\left(\Gamma_{\mathrm{v}}\right), \mathrm{e}\right| \operatorname{rel}\left(\Gamma_{\mathrm{v}}\right), e . \Gamma^{e} \cdot e^{-1}=\Gamma^{\bar{e}}, e \bar{e}=\delta_{e}^{e}, e=1$ if $\left.e \in E(\Lambda)\right\rangle$, where $\mathrm{v} \in \mathrm{V}(\mathrm{Z})$, and $\mathrm{e} \in \mathrm{E}(\mathrm{Z})$.

The notation $e . \Gamma^{e} \cdot e^{-1}=\Gamma^{\bar{e}}$ stands for the set of relations of the form $e \cdot a^{e} \cdot e^{-1}=\phi_{e}\left(a^{e}\right)=\left(\mu_{e}(a)\right)^{\bar{e}}, a \in \Gamma_{e}$.

$\pi \Phi(Z ; \Gamma ; \Lambda)$ is called the fundamental group of $\Phi(Z, \Gamma)$ relative to $\Lambda$.

For each e $\in \mathrm{E}(Z)$, let $t_{e}$ be the value of e in $\pi \Phi(Z ; \Gamma ; \Lambda)$ where no confusion will be caused by the notations $t_{e}$ and $\mathrm{t}(\mathrm{e})$. It is clear that the relations of $\pi \Phi(\mathrm{Z} ; \Gamma ; \Lambda)$ imply the following.

(1) $\pi \Phi(Z ; \Gamma ; \Lambda)$ is generated by elements $t_{e}$ and $g$, where $g \in \Gamma_{v}$ and $\mathrm{v} \in \mathrm{V}(\mathrm{Z})$;

(2) $t_{e} \notin \Gamma_{v}, \mathrm{v} \in \mathrm{V}(\mathrm{Z})$;

(3) $t_{e}=1$ if $\mathrm{e} \in \mathrm{E}(\Lambda)$; 
(4) $t_{e} g^{e} t_{e}^{-1}=\left(\phi_{e}(g)\right)^{\bar{e}}, \mathrm{~g} \in \Gamma^{e}$;

(5) $t_{e} t_{\bar{e}}=\delta_{e}^{e}$.

It is proved in [4, p. 148, 5.7 Corollary] that if $\Lambda_{1}$ and $\Lambda_{2}$ are two maximal subtrees of $Z$ then $\pi \Phi\left(Z ; \Gamma ; \Lambda_{1}\right)$ and $\pi \Phi\left(Z ; \Gamma ; \Lambda_{2}\right)$ are isomorphic.

The fundamental group of $\Phi(Z, \Gamma)$ denoted $\pi \Phi(Z ; \Gamma)$ is defined to be the fundamental group $\pi \Phi(Z ; \Gamma ; \Lambda)$ relative to a maximal subtree $\Lambda$ of $Z$.

In view of above, $\pi \Phi(Z ; \Gamma)$ is independent of any maximal subtree of $Z$.

The main result of this section is the following theorem.

Theorem 3.1. Let $G$ be a group acting on a connected quasi-graph $X$ with inversions, and $(\mathrm{Y} ; \mathrm{T})$ be a fundamental domain for the action of $\mathrm{G}$ on $\mathrm{X}$.

For each $\mathrm{v} \in \mathrm{V}(\mathrm{T})$ and $\mathrm{e} \in \mathrm{E}(\mathrm{Y})$, let $\Gamma_{G(v)}=G_{v}$ and $\Gamma_{G(e)}=G_{e}$.

Then $\Phi=(G /(X ; Y ; T) ; \Gamma)$ forms a quasi-graph of groups, and the fundamental group $\pi(\Phi)=\pi((G /(X ; Y ; T) ; \Gamma))$ has the presentation

$\left\langle\operatorname{gen}\left(G_{\mathrm{v}}\right), \mathrm{e}\right| \operatorname{rel}\left(G_{\mathrm{v}}\right), e \cdot G_{+(\bar{e})} \cdot e^{-1}=G_{+e}, e \bar{e}=\partial_{e}, e=1$ if $\left.\left.e \in E(T)\right\rangle \ldots{ }^{*}\right)$

where $\mathrm{v} \in \mathrm{V}(\mathrm{T})$, and $\mathrm{e} \in \mathrm{E}(\mathrm{Y})$. Furthermore, if $\mathrm{X}$ is a tree, then $\mathrm{G}$ and $\pi(\Phi)=\pi((G /(X ; Y ; T) ; \Gamma))$ are isomorphic.

Proof. By Lemma $2.1, G /(X ; Y ; T)$ forms a connected quasi-graph. Now we show that $\Gamma$ satisfies the conditions of the definition of quasi-graph of groups.

If $\mathrm{e}$ is an edge of $\mathrm{Y}$, then $\mathrm{G}(\mathrm{e})$ is an edge of $G /(X ; Y ; T)$.

(1) Now we show that $\Gamma_{\overline{G(e)}}=\Gamma_{G(e)}$. The fact that $G_{e}=G_{\bar{e}}$ implies that $\Gamma_{\overline{G(e)}}=\Gamma_{G(e)}$.

(2) We need to find a monomorphism $\lambda_{G(e)}: \Gamma_{G(e)} \rightarrow \Gamma_{t(G(e))}$.

We have $\mathrm{t}(\mathrm{e})=[\mathrm{e}]\left((\mathrm{t}(\mathrm{e}))^{*}\right)$. This implies that $G_{t(e)}=G_{[e](t(e) 8)}=[e] G_{(t(e))^{*}}[e]^{-1}$. Then

$\mathrm{t}(\mathrm{G}(\mathrm{e}))=\mathrm{G}(\mathrm{t}(\mathrm{e})), \quad \Gamma_{G(e)}=G_{e} \leq G_{t(e)}=[e] G_{(t(e))^{*}}[e]^{-1}, \quad \Gamma_{t(G(e))}=\Gamma_{G(t(e))}=G_{(t(e))^{*}}=$ $=[e]^{-1} G_{t(e)}[e]$, and $\Gamma_{o(G(e))}=\Gamma_{G(o(e))}=G_{o(e)}=G_{t(\bar{e})}=[\bar{e}]^{-1} G_{t(\bar{e})}[\bar{e}]=[e] G_{o(e)}[e]^{-1}$.

This implies that the mapping $\lambda_{G(e)}: G_{e} \rightarrow G_{t(e)}$ given by

$\lambda_{G(e)}(g)= \begin{cases}g & \text { if } t(e) \in V(T) \\ {[e]^{-1} g[e]} & \text { if } o(e) \in V(T)\end{cases}$

yields the required monomorphism $\lambda_{G(e)}: \Gamma_{G(e)} \rightarrow \Gamma_{t(G(e))}$, and similarly for the mapping $\lambda_{\overline{G(e)}}=\lambda_{G(\bar{e})}: G_{e} \rightarrow G_{o(e)}$ given by $\lambda_{G(\bar{e})}(g)= \begin{cases}{[e] g[e]^{-1}} & \text { if } t(e) \in V(T) \\ g & \text { if } o(e) \in V(T)\end{cases}$ is the required monomorphism $\lambda_{G(\bar{e})}: \Gamma_{G(e)} \rightarrow \Gamma_{o(G(e))}$. 
(3) Let $\delta_{G(e)}=[e][\bar{e}]$. If $\mathrm{G}(\mathrm{e}) \neq \mathrm{G}(\bar{e})$, then $[e]=[\bar{e}]^{-1}$, and $\delta_{G(e)}=1$, and we take $\mu_{G(e)}: G_{e} \rightarrow G_{e}$ to be the identity automorphism.

If $\mathrm{G}(\mathrm{e})=\mathrm{G}(\bar{e})$, then $\delta_{G(e)}=[e]^{2}$.

Let $\mu_{G(e)}: G_{e} \rightarrow G_{e}$ be the mapping given by $\mu_{G(e)}(g)=[e] g[e]^{-1}$.

It is easy to show that $\mu_{G(e)}$ is an automorphism. Furthermore, $\mu_{G(e)}\left(\delta_{G(e)}\right)=\mu_{G(e)}\left([e]^{2}\right)=[e][e]^{2}[e]^{-1}=[e]^{2}=\delta_{G(e)}$, and for $\mathrm{g} \in G_{e}$ we have $\left(\mu_{G(e)}\right)^{2}(g)=\mu_{G(e)}\left([e] g[e]^{-1}\right)=[e]^{2} g[e]^{-2}=\delta_{G(e)} g \delta_{G(e)}^{-1}$.

This implies that the mapping $\mu_{G(e)}: \Gamma_{G(e)} \rightarrow \Gamma_{G(e)}$ is the required automorphism. This implies that $\Phi=(G /(X ; Y ; T) ; \Gamma)$ forms a quasi-graph of groups.

Let e be an edge of $Y$. Then

$$
\begin{aligned}
\Gamma^{G(e)}=\lambda_{G(e)}\left(\Gamma_{G(e)}\right)=\lambda_{G(e)}\left(G_{e}\right) & =\left\{\begin{array}{ll}
G_{e} & \text { if } t(e) \in V(T) \\
{[e]^{-1} G_{e}[e]} & \text { if } o(e) \in V(T)
\end{array} \quad G_{+(\bar{e})},\right.
\end{aligned}
$$

and $\Gamma^{\overline{G(e)}}=\Gamma^{G(\bar{e})}=G_{+e}$. Furthermore, the mapping $\phi_{G(e)}: \Gamma^{G(e)} \rightarrow \Gamma^{\overline{G(e)}}$ given by $\phi_{G(e)}(\mathrm{g})=[\mathrm{e}] \mathrm{g}[e]^{-1}$ is an isomorphism. Furthermore,

$\phi_{G(e)}\left(\delta_{\tilde{e}}^{\tilde{e}}\right)=\phi_{G(e)}\left([e]^{2}\right)=[\mathrm{e}][e]^{2}[e]^{-1}=[e]^{2}$.

$G /(X ; T ; T)=\{G(v): v \in V(T)\} \cup\{G(e): e \in E(T)\}$. In the presentation (*), we replace $\Gamma_{v}$ by $G_{v}, \Gamma^{G(e)}$ by $G_{+(\bar{e})}, \Gamma^{\overline{G(e)}}$ by $G_{+e}$, and $\delta_{\tilde{e}}^{\tilde{e}}$ by $=\partial_{e}$. This leads the presentation of the fundamental group $\pi(\Phi)=\pi((G /(X ; Y ; T) ; \quad \Gamma))$ of $\Phi=(G /(X ; Y ; T)$ relative to the maximal subtree $G /(X ; T ; T)$ of $G /(X ; Y ; T)$.

If $\mathrm{X}$ is a tree, by [8, Corollary 5.2], $\mathrm{G}$ has above presentation. This implies that $\mathrm{G}$ and $\pi(\Phi)=\pi((G /(X ; Y ; T) ; \Gamma))$ are isomorphic. This completes the proof.

\section{On Subgroups of Quasi-Graph Groups}

Recall that a group $\mathrm{G}$ is called a quasi-graph group if there exists a quasi-graph of groups $\Phi=(Z ; \Gamma)$ where $Z$ contains more than one vertex such that $G=\pi(\Phi)$. The main result of this section is the following theorem.

Theorem 4.1. A subgroup of a quasi-graph group is a quasi-graph group. 
Proof. Let $G$ be a fundamental group of a quasi-graph of groups $\Phi(Z, \Gamma)$, where $\mathrm{Z}$ contains more than one vertex, $\Lambda$ be a maximal subtree of $\mathrm{Z}$, and $\mathrm{H}$ be a subgroup of $\mathrm{G}$. We need to find a quasi-graph of groups $\Phi_{H}=\left(Z_{H} ; \Gamma_{H}\right)$, where $\mathrm{H}$ is its fundamental group. In [5], a tree $X$ is constructed as follows. $\mathrm{V}(\mathrm{X})=\{[\mathrm{g}, \mathrm{v}]: \mathrm{v} \in \mathrm{V}(\mathrm{Z}), \mathrm{g} \in \mathrm{G}\}$, and $\mathrm{E}(\mathrm{X})=\{[\mathrm{g}, \mathrm{e}]: \mathrm{e} \in \mathrm{E}(\mathrm{Z}), \mathrm{g} \in \mathrm{G}\}$, where $[\mathrm{g}, \mathrm{v}]=\left(\mathrm{g} \Gamma_{v}, \mathrm{v}\right)$, and $[\mathrm{g}, \mathrm{e}]=\left(\mathrm{g} \Gamma^{e}, \mathrm{e}\right)$. The inverse and the terminals of the edge $[\mathrm{g}, \mathrm{e}]$ are defined as follows. $\overline{[g, e]}=\left[g t_{\bar{e}}, \bar{e}\right], \quad o[g, e]=\left[g t_{\bar{e}}, o(e)\right]$, and $t[g, e]=[g, t(e)]$. For $\mathrm{f} \in \mathrm{G}, \mathrm{v} \in \mathrm{V}(\mathrm{Z})$, and $\mathrm{e} \in \mathrm{E}(\mathrm{Z})$, define $\mathrm{f}[\mathrm{g}, \mathrm{v}]=[\mathrm{fg}, \mathrm{v}]$, and $\mathrm{f}[\mathrm{g}, \mathrm{e}]=[\mathrm{fg}, \mathrm{e}]$. If $\bar{e}=e$, then $\overline{[1, e]}=\left[t_{\bar{e}}, \bar{e}\right]=\left[t_{e}, e\right]=t_{e}[1, e]$. This implies that $\mathrm{G}$ acts on $\mathrm{X}$ with inversions. The $\mathrm{G}$ stabilizers of $[\mathrm{g}, \mathrm{v}]$ and $[\mathrm{g}, \mathrm{e}]$ are $G_{[g, v]}=g \Gamma_{\nu} g^{-1}$, and $G_{[g, e]}=g \Gamma^{e} g^{-1}$. The tree of representatives $\mathrm{T}$ for the action on of $\mathrm{G}$ on $\mathrm{X}$ is defined as $\mathrm{V}(\mathrm{T})=\{[1, \mathrm{v}]: \mathrm{v} \in \mathrm{V}(\mathrm{Z})\}$, and $\mathrm{E}(T)=\{[1, \mathrm{e}]: \mathrm{e} \in \mathrm{E}(\Lambda)\}$. Also, $[\mathrm{g}, \mathrm{v}]^{*}=[1, \mathrm{v}]$. The transversal $\mathrm{Y}$ for the action on of $\mathrm{G}$ on $\mathrm{X}$ consists of all edges of the form $\left[t_{e}, e\right], \mathrm{e} \in \mathrm{E}(\mathrm{X})$, with addition to their inverses $\overline{\left[t_{e}, e\right]}=[1, \bar{e}]$, and their terminals $o\left[t_{e}, e\right]=[1, o(e)]$, and $t\left[t_{e}, e\right]=\left[t_{e}, t(e)\right]$. Then $(\mathrm{T} ; \mathrm{Y})$ is a fundamental domain for the action of $\mathrm{G}$ on $\mathrm{X}$.

For each vertex $v \in \mathrm{V}(\mathrm{T})$, and each edge $\mathrm{e} \in \mathrm{E}(\mathrm{Y})$, define the following.

(a) $D_{v}$ is a double coset representative system for $\mathrm{G} \bmod \left(\mathrm{H}, G_{v}\right)$,

(b) $D_{e}^{g}$ and $D_{\bar{e}}^{g}$ are any double coset representative systems for $G_{o(e)} \bmod \left(G_{o(e)} \cap g^{-1} H g, G_{e}\right)$, and $G_{(t(e))^{*}} \bmod \left(G_{(t(e))^{*}} \cap g^{-1} H g,[e]^{-1} G_{y}[e]\right)$

respectively containing 1 , but otherwise arbitrary, $g \in \mathrm{G}$. For more details of the structures of $D_{v}, D_{e}^{g}$ and $D_{\bar{e}}^{g}$ we refer the readers to [7].

The fundamental domain $\left(T_{H}, Y_{H}\right)$ for the action of $\mathrm{H}$ on $\mathrm{X}$ obtained in [7] is defined as follows. $\mathrm{V}\left(T_{H}\right)=\left\{\mathrm{d}(\mathrm{v}): \mathrm{v} \in \mathrm{V}(\mathrm{T}), \mathrm{d} \in D_{v}\right\}$, and $\mathrm{E}\left(T_{H}\right)$ is the set of edges, $\left\{\mathrm{ab}(\mathrm{e}), \mathrm{ab}(\bar{e}): \mathrm{e} \in E_{0}(\mathrm{Y}) \cup E_{1}(\mathrm{Y}) \cup E_{2}(\mathrm{Y}), \mathrm{a} \in D_{o(e)}, \mathrm{b} \in D_{e}^{a}, \mathrm{ab} \in D_{(t(e))^{*}}\right\}$, where $E_{0}(\mathrm{Y})=\left\{\left[t_{e}, e\right]=[1, \mathrm{e}]: \mathrm{e} \in \mathrm{E}(\Lambda)\right\} ; E_{1}(\mathrm{Y})=\left\{\left[t_{e}, e\right]: \mathrm{e} \in \mathrm{A}, \bar{e} \neq e\right\}$, and $E_{2}(\mathrm{Y})=\left\{\left[t_{e}, e\right]: \mathrm{e} \in \mathrm{A}, \bar{e}=e\right\}$.

$Y_{H}$ consists of the edges of the forms $\mathrm{ab}(\mathrm{e})$, and $\mathrm{ab}(\bar{e})$, where $\mathrm{e} \in E_{0}(\mathrm{Y}) \cup E_{1}(\mathrm{Y}) \cup E_{2}(\mathrm{Y}), \mathrm{a} \in D_{o(e)}$ and $\mathrm{b} \in D_{e}^{a}$, and their terminals.

Similar to the sets of edges $E_{0}(\mathrm{Y}), E_{1}(\mathrm{Y})$, and $E_{2}(\mathrm{Y})$, the structures of the sets of the edges $E_{0}\left(Y_{H}\right), E_{1}\left(Y_{H}\right)$, and $E_{2}\left(Y_{H}\right)$ can be formed as follows. $E_{0}\left(Y_{H}\right)=\mathrm{E}\left(T_{H}\right)$ consists of the set of edges of the following forms. 
(a) $\mathrm{ab}(\mathrm{m})$, where $\mathrm{a} \in D_{o(m)}$, and $\mathrm{b} \in D_{m}^{a}$ such that $\mathrm{ab} \in D_{t(m)}$;

(b) $\mathrm{ab}(\mathrm{y})$, where $\mathrm{a} \in D_{o(y)}$, and $\mathrm{b} \in D_{y}^{a}$ such that $\mathrm{ab}[\mathrm{y}] \in D_{(t(y))^{*}}$;

(c) $\mathrm{ab}(\mathrm{x})$, where $\mathrm{a} \in D_{o(x)}$ and $\mathrm{b} \in D_{x}^{a}$ such that $\mathrm{ab}[\mathrm{x}] \in D_{o(x)}$ and

$\mathrm{H} \cap a b[x] G_{x} b^{-1} a^{-1}=\varnothing$.

$E_{1}\left(Y_{H}\right)$ consists of the set of edges of the following forms.

(a) $\mathrm{ab}(\mathrm{m})$, where $\mathrm{a} \in D_{o(m)}$ and $\mathrm{b} \in D_{m}^{a}$ such that $\mathrm{ab} \notin D_{t(m)}$;

(b) $\mathrm{ab}(\mathrm{y})$, where $\mathrm{a} \in D_{o(y)}$, and $\mathrm{b} \in D_{y}^{a}$ such that $\mathrm{ab}[\mathrm{y}] \notin D_{(t(y))^{*}}$;

(c) $\mathrm{ab}(\mathrm{x})$, where $\mathrm{a} \in D_{o(x)}$ and $\mathrm{b} \in D_{x}^{a}$ such that $\mathrm{ab} \quad[\mathrm{x}] \notin D_{o(x)}$ and $\mathrm{H} \cap a b[x] G_{x} b^{-1} a^{-1}=\varnothing$.

$E_{2}\left(Y_{H}\right)$ consists of the set of edges of the form: $\mathrm{ab}(\mathrm{x})$, where $\mathrm{a} \in D_{o(x)}$, and $\mathrm{b} \in D_{x}^{a}$ such that $\mathrm{ab}[\mathrm{x}] \notin D_{o(x)}$, and $\mathrm{H} \cap a b[x] G_{x} b^{-1} a^{-1} \neq \varnothing$.

Theorem 3.1 implies that $\mathrm{H}$ is the fundamental group of the quasi-graph of groups $\Phi_{H}=\left(H /\left(X ; Y_{H} ; T_{H}\right) ; \Gamma\right)$. Consequently $\mathrm{H}$ is a quasi-graph group. This completes the proof.

\section{References}

[1] H. Bass, Covering theory for graphs of groups, J. Pure Appl, 89, No. 1-2, 3-47(1993).

[2] W. Dicks and M. J. Dunwoody, Groups acting on graphs, Cambridge University Press (1989).

[3] M. I. Khanfar and R. M. S. Mahmud, A note on groups acting on connected graphs, J. Univ. Kuwait Sci. 16 no. 2, 205-208(1989).

[4] R. M. S. Mahmood, Fundamental Groups of Quasi Graphs of Groups, Hacettepe Journal of Mathematics and Statistics. Volume 39 (2), 137-149 (2010).

[5] R. M. S. Mahmood, On trees induced by quasi graphs of groups, Algebras Groups Geom. 25, No. 3, 267-285 (2008).

[6] R. M. S. Mahmood, On quasi graphs of groups induced by groups acting on trees with inversions, Hadronic J. 30 no. 6, 639-655(2007). 
[7] R. M. S. Mahmood, The subgroup theorem for groups acting on trees, Kuwait J. Sci. Engrg, 25, no. 1, 17-33 (1998).

[8] R.M.S. Mahmud, Presentation of groups acting on trees with inversions, Proc. R. Soc. Edinb., Sect. A , 113 No.3-4, 235-241(1989).

[9] J-P. Serre, Trees, Translated by John Stillwell, Springer-Verlag (1980).

Received: January 5, 2014 